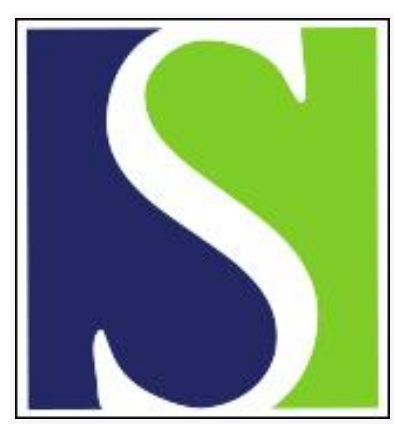

Scand J Work Environ Health 2007;33(6):401-404

https://doi.org/10.5271/sjweh.1161

Issue date: 31 Dec 2007

Physical activity and cardiovascular health-work and leisure differ

by Kukkonen-Harjula K

Affiliation: UKK Institute for Health Promotion Research, PO Box 30, Fl-33501 Tampere, Finland. katriina.kukkonen-harjula@uta.fi

Refers to the following texts of the Journal: 2003;29(5):363-377

2007;33(6):405-424 2007;33(6):425-434 2002;28(2):73-74

The following articles refer to this text: 2010;36(5):349-355;

2019;45(3):267-279

Key terms: cardiovascular health; editorial; leisure; physical activity; work

This article in PubMed: www.ncbi.nlm.nih.gov/pubmed/18327508 


\section{Physical activity and cardiovascular health—work and leisure differ}

During the last century, worklife has changed a great deal, and, as a result, energetic demands have decreased in the workplace. For more than half a century, researchers in cardiovascular epidemiology have studied cardiovascular load at work and its relation to cardiovascular disease (CVD). However, occupational physical activity still accounts for a considerable proportion of all physical activity, the estimations being around $30 \%$ for developed countries. In addition, a considerable proportion of working people is exposed to high energetic demands at work $(1,2)$ (eg, in the construction and metal industries and in agriculture). Thus, when musculoskeletal strain, new demands from information technology, and work stress are taken into consideration, it seems that the overall strain may not have decreased markedly in all jobs.

In this issue of the Scandinavian Journal of Work, Environment \& Health, Krause et al (3) report a study carried out with a representative sample of 612 middle-aged Finnish men, of whom nearly a third were exposed to energetic demands that exceeded the upper level of recommended aerobic strain. The report highlights the association between occupational physical activity and the progression of atherosclerosis in an 11-year follow-up. Occupational physical activity was defined by estimating energy expenditure in three interviews. Energy expenditure was also adjusted for baseline aerobic fitness, which was assessed by the direct measurement of maximal oxygen consumption. The study thus included elaborate and repeated measures of energy expenditure, mainly describing aerobic activities. Repeated ultrasound assessments were performed to define the carotid intima-media thickness (IMT), which is a reliable surrogate marker of coronary atherosclerosis and which predicts coronary heart disease (CHD) events. The main outcome was the change in IMT (ie, the progression of atherosclerosis). The validity of sonography in predicting future cardiovascular events has recently been reviewed by Bots et al (4) and found to be acceptable.

The main finding of Krause et al's study (3) was that high energy consumption was positively associated with an accelerated progression of atherosclerosis after control for several CVD risk factors, including job factors related to work stress and leisure-time physical activity. CHD, carotid plaque, or stenosis at baseline enhanced the progression of atherosclerosis in combination with high energy consumption at work. It remains to be seen how many new CHD cases will be found in the follow-up. As practical implications for occupational health care, Krause et al (3) suggested that the relative aerobic strain of new workers, or in cases of new CVD diagnosis, should be assessed in jobs with known high aerobic strain. For this purpose, occupational health care needs equipment for carrying out the measurements and expertise in interpreting the results. However, overall CVD risk assessment, musculoskeletal strain, and work stress should not be forgotten.

Another report (5) in the current issue of the Scandinavian Journal of Work, Environment \& Health also concentrates on high physical workload, both alone and in combination with two other exposures, noise and shift work. The outcome of the study by Virkkunen et al (5) was the incidence of coronary events in an 8-year follow-up of both blue- and white-collar workers in industry. These authors studied how health effects were modulated through increased systolic blood pressure. The classification of physical workload was based on occupational coding and not on individual questionnaires. The main finding was that the elevation of systolic blood pressure was associated with high workload, regardless of other exposures, that led to increased CHD risk. 
The long-term follow-up cohorts of Krause et al (3) and Virkkunen et al (5) provide further evidence that high energy consumption at work may have detrimental effects on cardiovascular health. This finding is contrary to strong epidemiologic and experimental evidence indicating that low physical activity is an independent risk factor for CVD, especially atherosclerosis (6). However, most of the newest data are based on physical activity during leisure time and not on physical activity at work. Thus the resulting physiological effects of high energetic demands at work on physical fitness and work capacity are not similar to those due to leisure-time exercise training. The most obvious reason is that high energetic demands at work continue for hours in comparison with much shorter bouts of physical activity during leisure time. In addition, both the ability to control the task and psychological expectations very often differ.

Could worksite physical activity programs be of help in alleviating the detrimental effects of high energy demands at work? Actually this possibility has not been specifically studied, and reviews on worksite physical activity programs do not provide straightforward answers about how workers' health and productivity should be promoted (7). In cases of high energetic demand at work, exercise programs should be performed during leisure time, while work breaks should be used for rest and eventual muscle stretching and relaxation.

While work physiology and occupational medicine both concentrate on energy consumption (aerobic strain) at work, research on physical activity in sports medicine has focused on leisure-time activities, including structured exercise training, various household activities, and commuting to and from work in a physically active manner. As people's worklife has changed, their leisure-time physical activity has often decreased, even if the available time for leisure-time activities may have increased in the long term. A sedentary lifestyle (ie, low physical activity) is a risk factor for many diseases. According to systematic reviews $(8,9)$ based on evidence from both observational cohorts and randomized controlled exercise training trials, mainly during leisure time, regular physical activity results in reduced all-cause mortality, a reduced incidence of fatal and nonfatal total CVD and CHD, a reduced incidence of obesity and type2 diabetes, improved metabolic control of type-2 diabetes, a reduced incidence of colon cancer and osteoporosis, improved physical function and independent living among the elderly, less likelihood of depression and improvements in symptoms of depression and anxiety, and favorable impacts on CVD risk factors.

Therefore, exercise recommendations for adult populations [eg, the newest ones from the American College of Sports Medicine and the American Heart Association $(10,11)]$ especially concentrate on increasing moderate-intensity physical activity during leisure time. Moderate-intensity aerobic exercise means 3-6 MET (metabolic equivalents, a multiple of resting energy consumption). Accordingly, healthy adults between the ages of 18 and 65 years should perform at least moderate-intensity aerobic (endurance) physical activity for a minimum of 30 minutes 5 days a week or vigorous-intensity (>6 METs) aerobic activity for a minimum of 20 minutes 3 days a week (7). In addition, people benefit from performing activities requiring the use of major muscle groups to increase or maintain muscular strength and endurance at least twice a week. Adults aged 50-64 years with chronic health conditions or functional limitations should follow recommendations for persons aged 65 years and over (11).

Currently about one-third of Finnish adults engage in leisure-time physical activity according to the aforementioned recommendations on health-enhancing physical activity. In addition to structured exercise training, a physically active lifestyle is another alternative. However, not all domestic physical activity is enough to improve cardiovascular fitness (12) because it generally represents mild-to-moderate intensity. Even though household activities can subjectively be perceived as heavy housework, they often involve small muscle groups in the upper body, in contrast to the large muscle groups employed in walking, running, and other endurance exercise modes.

Commuting to and from work in a physically active manner (eg, walking or cycling) can also offer people an opportunity to increase physical activity. Interest in commuting physical activity to improve 
physical fitness and work capacity has only increased during the last couple of decades. The main obstacles in the promotion of commuting physical activity may be environmental factors (eg, distance between the workplace and home) and the nature of work necessitating motorized transport. However, in Finland, about $50 \%$ of employed adults report at least some commuting physical activity daily, the frequency and duration being higher among women. A protective effect of commuting physical activity against CHD was found for women, but not for men, in a 19-year follow-up of more than 47000 adults (13). When all physical activity, either at work, during commuting or during leisure time, was considered, the protective effect of leisure-time physical activity was only found for men with low occupational physical activity, while the effect was observed for women with any level of occupational physical activity. This is the most important conclusion of the study, since, even in the best of situations, commuting physical activity is much shorter in duration than other types of leisure-time physical activity.

In developed countries, the sector of the working population over 50 years of age will grow considerably in the near future. After the year 2010, the number of retired people over 65 years of age will be almost double that of 1995, the probable result being a strong impact on work conditions and on the labor market. Many people will still be working in physically demanding occupations. Thus there is still a need to decrease high energetic demands through work design and, in cases of work with high demands, to assess individual worker's physical fitness for their tasks. We also need further research and developmental work on how to promote physically active commuting to work and how to organize health-enhancing physical activity in jobs with irregular workhours. Counseling on other lifestyle aspects through occupational health care should be combined with physical activity (eg, dietary changes in cases of obesity and dyslipidemias) or improvement in sleep hygiene in cases of poor sleep. It is also important to study what the combined effects of lifestyle changes are. A major challenge in occupational health care is to develop feasible counseling methods to prevent lifestyle diseases, as we are facing the epidemic of obesity and its comorbidities. In conclusion, occupational physical activity could form an integral part of habitual physical activity and could result in improved health rather than in detrimental effects, providing the intensity, duration, frequency, and total volume of the physical demands and rest during the workday are suitable for the worker.

\section{References}

1. Karlqvist LK, Härenstam A, Leijon O, Schéele P; MOA Research Group. Excessive physical demands in modern worklife and characteristics of work and living conditions of persons at risk. Scand J Work Environ Health. 2003;29(5):363-77.

2. Proper KI, Hildebrandt VH. Physical activity among Dutch workers-differences between occupations. Prev Med. 2006;43(1):42-5.

3. Krause N, Brand RJ, Kaplan GA, Kauhanen J, Malla S, Tuomainen TP, et al. Occupational physical activity, energy expenditure, and 11-year progression of carotid atherosclerosis. Scand J Work Environ Health. 2007;33(6):405-24.

4. Bots ML, Baldassarre D, Simon A, de Groot E, O'Leary DH, Riley W, et al. Carotid intima-media thickness and coronary atherosclerosis: weak or strong relations? Eur Heart J. 2007;28(4):398-406.

5. Virkkunen H, Härmä M, Kauppinen T, Tenkanen L. Shift work, occupational noise and physical workload with ensuing development of blood pressure and their joint effect on risk coronary heart disease. Scand J Work Environ Health. 2007;33(6):425-34.

6. Kohl HW 3rd. Physical activity and cardiovascular disease: evidence for a dose response. Med Sci Sports Exerc. 2001;33(6 Suppl):S472-83.

7. Härmä M. Worksite physical activity—a useful, but not sufficient action for promoting work-related health and productivity [editorial]. Scand J Work Environ Health. 2002;28(2):73-4.

8. Dose-response issues concerning physical activity and health: an evidence-based symposium. Med Sci Sports Exerc. 2001;33(6 Suppl):S345-641.

9. Bauman AE. Updating the evidence that physical activity is good for health: an epidemiological review 2000-2003. J Sci Med Sport. 2004;7(1 Suppl):6-19.

10. Haskell WL, Lee IM, Pate RR, Powell KE, Blair SN, Franklin BA, et al. Physical activity and public health: updated recommendation for adults from the American College of Sports Medicine and the American Heart Association. Circulation. 2007;116(9):1081-93.

11. Nelson ME, Rejeski WJ, Blair SN, Duncan PW, Judge JO, King AC, et al. Physical activity and public health in older 
adults: recommendation from the American College of Sports Medicine and the American Heart Association. Circulation. 2007;116(9):1094-105.

12. Stamatakis E, Hillsdon M, Primatesta P. Domestic physical activity in relationship to multiple CVD risk factors. Am J Prev Med. 2007;32(4):320-7.

13. Hu G, Jousilahti P, Borodulin K, Barengo NC, Lakka TA, Nissinen A, et al. Occupational, commuting and leisure-time physical activity in relation to coronary heart disease among middle-aged Finnish men and women. Atherosclerosis. 2007;194(2):490-7.

Katriina Kukkonen-Harjula, MD

UKK Institute for Health Promotion Research

PO Box 30

FI-33501 Tampere, Finland

[katriina.kukkonen-harjula@uta.fi] 